\section{ÜBRIGENS}

.... war ein hochintensives Intervalltraining bei Patienten mit Herzinsuffizienz in der SMARTEX-HF-Studie wider Erwarten nicht effektiver als ein Ausdauertraining von moderater Intensität.

.... ist ein primärer Hyperaldosteronismus als Hypertonie-Ursache keine Seltenheit. In einer italienischen Studie wurde diese Ursache bei immerhin 6\% aller in Praxen betreuten Patienten mit Bluthochdruck nachgewiesen. Ein Screening könnte sich also lohnen.

\section{Therapie der Aortenstenose}

\section{TAVI bleibt auf Erfolgskurs}

Als Behandlungsmethode bei Patienten mit schwerer symptomatischer Aortenstenose hat die perkutane Transkatheter-Aortenklappen-Implantation (TAVI) den chirurgischen Aortenklappenersatz in Deutschland inzwischen zahlenmäßig überflügelt. Zugleich werden die damit erzielten Behandlungsergebnisse immer besser. So lag die Krankenhaussterblichkeit nach transvaskulärer TAVI 2015 nur noch bei 3,4\%. Im Vergleich dazu lag sie 2014 bei 3,8\%. Im Jahr 2013 hatte die Mortalitätsrate nach TAVI noch 8,3\% betragen.

Punkte sammeln in der Sommerakademie mit e.Med

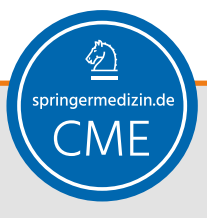

Auch 2017 haben wir in der Sommerakademie ausgewählte CME-Kurse für Ihre hausärztliche Fortbildung zusammengestellt.

Zum Thema "Kardiologie" finden Sie unter

http://bit.ly/2hFwgdK

aktuell den Kurs

\section{„Diabetes und Herzinsuffizienz -}

\section{Update 2017"}

Zur Teilnahme benötigen Sie ein e.Med-Abo, mit dem Sie Zugang zu allen Inhalten auf SpringerMedizin.de erhalten.

Nutzen Sie die Sommerzeit, um CME-Punkte zu sammeln und um sich schnell und effizient fortzubilden.

Testen Sie SpringerMedizin.de 30 Tage lang kostenlos und unverbindlich mit dem e.Med-Abo: http://bit.ly/2vhm8el

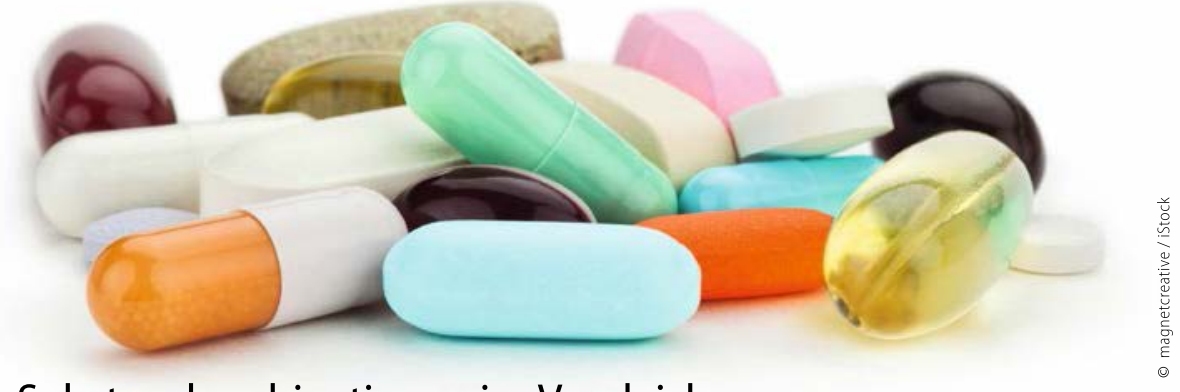

Substanzkombinationen im Vergleich

\title{
Was ist die beste Therapie der Herzinsuffizienz?
}

Zur Prognoseverbesserung bei Herzinsuffizienz sind mehrere Optionen verfügbar. Welche Medikamente in welcher Kombination die Sterblichkeit am effektivsten senken, haben Forscher in einer Netzwerk-Metaanalyse untersucht.

Zur Therapie der Herzinsuffizienz mit reduzierter Auswurffraktion (HFrEF) können Ärzte heutzutage auf fünf verschiedene Substanzklassen zurückgreifen, die nachweislich die Sterblichkeit der Patienten senken: ACE-Hemmer, $\mathrm{AT}_{1^{-}}$ Rezeptorantagonisten (ARB), Betablocker, Mineralkortikoid-Rezeptorantagonisten (MRA) und seit neuestem auch ein Angiotensin-Rezeptor-Neprilysin-Inhibitor, kurz ARNI.

Wie effektiv die einzelnen Substanzen in Relation zu den anderen sind, ist unklar, da es keine direkten Vergleichsstudien gibt. So wurden neuere Substanzen, in der Regel additiv zur üblichen Standardtherapie, geprüft; etwa im Falle des ARNI Sacubitril/Valsartan in der PARADIGM-HFStudie gegen einen ACE-Hemmer oder ARB in Kombination mit einem Betablocker und ggf. einem MRA. Die Wirksamkeit von ARNI gegenüber Placebo ist somit nicht direkt untersucht worden.

Forscher um Heather Burnett haben deshalb auf ein statistisches Verfahren zurückgegriffen, welches es erlaubt, die Wirksamkeit zweier Substanzen indirekt zu vergleichen [1]. Mit dieser sogenannten Bayes'schen Netzwerk-Metaanalyse lässt sich etwa die Wirksamkeit einer Substanz A gegenüber einer Substanz B indirekt vergleichen, indem Vergleichsstudien zwischen der Substanz C und B und der Substanz $C$ und A herangezogen werden. Die Wissenschaftler erstellten so ein Netzwerk aus 57 randomisierten Studien, in denen die genannten Medikamente bzgl. ihrer Effektivität, die Sterblichkeit der HFrEF zu senken, verglichen worden sind.

\section{Kombinationstherapien effektiver}

Am effektivsten stellte sich hierbei die Kombination aus ARNI, Betablocker und MRA heraus: Die Sterblichkeit ließ sich damit im Vergleich zu Placebo um 63\% verringern. Die Kombi aus ACE-Hemmer, Betablocker und MRA ging mit einer Mortalitätsreduktion um $56 \%$ einher und belegte den zweiten Platz. Generell erwiesen sich die Kombinationstherapien als deutlich effektiver als die Einzelsubstanzen.

Damit bestätige diese Analyse die derzeit gültigen Leitlinienempfehlungen, resümieren die Studienautoren. Zur FirstLine-Therapie bei HFrEF gehört heute ein ACE-Hemmer (bei Unverträglichkeit: $\mathrm{AT}_{1}$-Blocker) in Kombination mit einem Betablocker. In der Zweitlinien-Therapie wird ein MRA dazu genommen. Bleiben die Patienten weiterhin symptomatisch, wird empfohlen, den ACE-Hemmer durch den ARNI zu ersetzen.

vsclob

1. Circ Heart Fail. 2017;10: e003529

\section{DATEN UND FAKTEN}

\subsection{8}

Patienten mit einer schweren Aortenstenose haben im Jahr 2015 in Deutschland eine Transkatheter-Aortenklappe auf transvaskulärem Weg - zumeist via transfemoralem Zugang erhalten. Nach Daten des IQTIG, dem Institut für Qualitätssicherung und Transparenz im Gesundheitswesen in Berlin, wurde die neue Aortenklappe in 9502 Fällen per isolierter (nicht mit koronarer Bypass-Chirurgie kombinierter) Klappenersatz-Operation implantiert. 